\title{
The Preparation of Coal based Graphene and It's Application in the Field of Thermal Conductivity
}

\author{
Wen-Wei Ma* \\ CCTEG Shenyang Research Institute, State Key Laboratory of Coal Mine Safety Technology, Fushun 113122, Liaoning, China
}

*Correspondence email: 120598723@qq.com

\begin{abstract}
Graphene is a new material with good physical properties and high added value. Coal can be made into model compounds by different physical and chemical methods to realize the efficient utilization of coal, so that new coal based materials with high added value can be formed. Based on this, in the process of research and analysis, the preparation of coal based graphene and its application in the field of thermal conductivity were studied. Based on the lateral guiding excitation of graphene, its performance and application of thermal conductivity are analyzed comprehensively. On the basis of external force control, it has a positive effect on improving the thermal conductivity of graphene composites.
\end{abstract}

Keywords

Coal based Graphene; Preparation; The field of Thermal Conductivity; Application Analysis

\section{INTRODUCTION}

Coal has the characteristics of high pollution, high energy consumption and low efficiency in actual application. Through processing, the added value of coal can be improved. If coal is processed into coal based new material products, its benefit will be relatively improved.Coal medium has the characteristics of loose structure and low price, which can be used in large-scale production of graphene. Based on this, the preparation and practical application of coal based graphene are studied, which has a positive role in improving the practical application effect of graphene. Graphene is a two-dimensional bee like planar structure formed by covalent bonds. The ultrathin two-dimensional planar structure improves the physical and chemical properties of graphene. Moreover, its thermal conductivity is relatively good, and its thermal conductivity can reach $5300 \mathrm{w} /(\mathrm{m} \bullet \mathrm{K})$. This paper focuses on the application of graphene in the field of thermal conductivity, which has a positive role in improving its application effect ${ }^{[1]}$. 


\section{PREPARATION AND ANALYSIS OF COAL BASED GRAPHENE}

\subsection{Analysis of Coal based Heat Treatment}

Raw coal has more impurities, so it can not be directly used in graphene. Therefore, raw coal needs to be purified. With the gradual deepening of coal metamorphism, the number of aromatic layers will increase, which can be chemically treated to obtain coal based aromatic materials. After the raw coal is crushed and screened, the powder with particle size less than $75 \mathrm{~mm}$ is obtained, and it is heated in a clamp pot at $1000^{\circ} \mathrm{C}$ for $2 \mathrm{hs}$. Volatile impurities can be removed at a heating rate of $5^{\circ} \mathrm{C}$ in the shielding gas argon. On this basis, the graphitization can be realized by heating at a heating rate of $10^{\circ} \mathrm{C} / \mathrm{min}$ to $2800^{\circ} \mathrm{C}$ in $2 \mathrm{hs}{ }^{[2]}$.

\subsection{Analysis of Preparation Methods of Coal based Graphene}

Raw coal is screened, impurities are removed and heat treatment is carried out to obtain high purity raw materials in the process of preparation and operation of coal based graphene. In the practical preparation process, it is often treated by oxidation-reduction method, chemical vapor deposition method and other methods. The degree of metamorphism is relatively high, the carbon content of coal is relatively high, and the content of fatty hydrocarbons and oxygen-containing functional groups and other impurities is relatively small. At high temperature, dehydrogenation and cyclization of aromatic structure will occur, which can gradually form large graphite crystals. Moreover, graphene can be obtained by oxidation-reduction ${ }^{[3]}$. In the study of coal with relatively low metamorphic degree, it contains a large number of aliphatic hydrocarbons and oxygen-containing functional groups, which can produce gaseous substances at low temperature, and can be prepared into coal cornerstone graphene by chemical vapor deposition. Taking coal as raw material, it is necessary to screen the raw coal, remove impurities in it at $1000^{\circ} \mathrm{C}$, and pyrolyze it at a temperature above $2000^{\circ} \mathrm{C}$, so as to obtain the raw material of coal based graphene in the process of preparation of coal based graphene. In the process of the preparation of graphene from coal, it needs to be treated in high temperature environment, which requires high equipment requirements, and its initial investment cost is relatively high. On this basis, protective gas should be filled to realize the preparation operation and control of coal based graphene in order to avoid oxidation in high temperature process ${ }^{[4]}$.

In the analysis of the factors affecting the preparation of coal based graphene, coal rank and macerals will have a direct impact on the preparation of coal based graphene. Bituminous coal contains many non aromatic structure and oxygen-containing groups, and its spatial structure is relatively loose and its porosity is relatively large, which has a direct impact on graphitization and preparation of graphene ${ }^{[5]}$. The results show that the oxygen-containing functional groups and alkyl side chains of ortho-bituminous coal are relatively small, the aromatic nuclei are relatively larger, and the bridge bonds between structural units are reduced. The overall structure of coal is relatively dense, and the porosity is relatively marginal, which is very easy to appear maximum or minimum value, which also has a direct impact on the preparation of coal cornerstone graphene. The condensation ring of high rank coal increased significantly. On the basis of enhanced macromolecular arrangement sequence, graphite structure can be formed, which is helpful for graphitization and preparation of coal based graphene. In the study of carbon content and composition changes, the component arrangement has a direct impact on the preparation of coal based graphene from the point of view of the micro components. There is a direct relationship between the chemical structure of macerals and the degree of coalification. Moreover, the difference of grouping is relatively small. In the research and analysis, we need to make a comprehensive analysis on the preparation process of coal based graphene and the changes of oxygen-containing functional groups, so as to further improve the preparation effect ${ }^{[6]}$.

\subsection{Thermal Conductivity Mechanism and Influencing Factors of Coal based Graphenel}

In the study of the thermal conductivity mechanism of graphene, graphene can conduct heat through phonons. In the realization of heat transfer, the low and high frequency of LA mode, TA mode and ZA mode are the main modes. In the process of heat transfer, La and TA can play a major role, while the role of ZA is 
relatively reduced. In the process of research and analysis, the contribution of ZA to the thermal conductivity of $10 \mu \mathrm{m}$ graphene is relatively low, accounting for about $20 \%$, and LA and TA accounting for $76 \%$. The temperature dependence of ZA is relatively strong. At T $<100 \mathrm{~K}$, ZA phonons mainly propagate by heat. In the case of $100 \mathrm{~K}<\mathrm{T}<200 \mathrm{~K}$, TA and LA phonons are the main transfer carriers. In the process of heat conduction and control, the thermal conductivity can be controlled to realize the thermal conductivity analysis and control of coal based graphene. In the process of analyzing the influencing factors of coal based graphene thermal conductivity, it is directly related to temperature, graphene size and defects. Graphene, in the single layer state, will be folding and agglomeration, this kind of graphene has no effective application value. The expression of heat conduction in phonon mode is as follows:

$$
\mathrm{K}=1 / 3 \mathrm{C}_{\mathrm{v}} \mathrm{vL}
$$

$\mathrm{K}$ is the heat transfer coefficient of graphene, $\mathrm{C}_{\mathrm{v}}$ is the specific heat capacity of phonons, $\mathrm{v}$ is the propagation velocity of phonons, and $\mathrm{L}$ is the average degree of freedom.

Graphene has a unique two-dimensional structure, which makes the sound velocity of phonons higher and higher. However, the specific heat and degree of freedom of graphene are greatly affected by temperature and graphene size. With the increase of temperature, the specific heat and degree of freedom of phonons will gradually increase, and the thermal conductivity will also increase. With the increase of temperature, the boundary size of phonon's degree of freedom will increase and its thermal conductivity will decrease. Based on this, it can be found that the size of graphene has an effect on the heat transfer of phonons. When the phonon space remains unchanged, graphene can pass through the hard acoustic barrier layer to improve the thermal conductivity ${ }^{[7]}$.

\section{APPLICATION ANALYSIS OF GRAPHENE IN THE FIELD OF THERMAL CONDUCTIVITY}

The contribution of graphene in the field of thermal conductivity is reflected in its thermal conductivity of oil, which is suitable for heat dissipation. The preparation of single-layer and two-dimensional graphene materials is relatively difficult, and its structure is not stable. Generally, a few layers of graphene and graphene are used as thermal conductive materials, and they are added to other materials, so as to improve the thermal conductivity of composite materials ${ }^{[8]}$. There are many functional groups in pure oxygen graphene. In the process of phonon transmission, it is easy to scatter, and the effect of heat transfer is not ideal. However, the combination of oxygencontaining functional groups and other materials can reduce the phonon heat dissipation, so as to further improve the heat transfer effect. The applications in the field of heat pipe mainly focus on minority layer graphene, graphene composites and graphene oxide composites. In the study of practical application of different graphene, there are some differences in the application effect ${ }^{[9]}$.

\subsection{Minority Layer Graphene}

Single layer graphene is the best thermal conductivity material among the known materials. It is easy to agglomerate and wrinkle. However, it is difficult to prepare a few layers of graphene. In the practical application of a few layers of graphene, the self heating of high-power GaN electrons and optoelectronic devices leads to the temperature rise, and the dissipation power distribution is uneven, which has a direct impact on the practical application of nitrogen-based technology ${ }^{[10]}$. When minority layer graphene are used in the field of heat conduction, it is necessary to analyze the heat dissipation effect of its CSG heat dissipation film. Under 5V voltage, the temperature decreases by $2.7^{\circ} \mathrm{C}$, and the thermal conductivity increases by $33 \%$. When the voltage is $12.5 \mathrm{~V}$, the core temperature decreases to $7.5^{\circ} \mathrm{C}$ and the thermal conductivity increases by $17 \%$. The thermal conductivity of minority layer graphene decreases, so it has a certain potential in the application of high performance heat dissipation components. However, the contact thermal resistance of minority layer graphene may appear in practical application, which affects the practical application range of a few graphene. 


\subsection{Application of Graphene Composites}

The traditional thermal interface material is based on polymer, oil and so on, and the filler is mainly composed of silver and silicon particles with large volume fraction $(>70 \%)$, so as to achieve the thermal conductivity of $4 \mathrm{~W} /(\mathrm{m} \bullet \mathrm{K})$. However, in practical application, fillers can easily cause the viscosity of thermal interface materials to decrease, and the actual production cost of composites will also increase. Graphene has excellent thermal conductivity in practical application. After adding a small amount of components, it can greatly improve the thermal conductivity of thermal interface materials without damaging the physical properties of interface materials, which can promote the production of heat dissipation materials for radiators. In the study of synchronous effect of graphene and silver powder, it can be added to silicone rubber, and its thermal conductivity can be improved, which shows that the application of graphene can improve the thermal conductivity of composite materials. In order to further increase the thermal conductivity of thermal interface materials, there are some differences in the practical application of graphene in the comprehensive analysis of metal or high thermal conductivity. Based on the comprehensive control of graphene, the thermal conductivity of graphene can be improved. Under different temperature perspectives, the thermal conductivity of graphene will also increase with the increase of temperature, which indicates that the thermal conductivity of thermal interface materials will also increase with the increase of heat generation of components, which indicates that graphene can be used as a heat dissipation material for thermal interface. With the increase of temperature, the thermal conductivity decreases with the increase of back scattering. In this context, the preparation and application of graphene composites do not take into account the specific thermal conductivity of graphene itself, but just randomly add graphene to the thermal interface materials. Based on this, it is necessary to make use of the high thermal conductivity of graphene and prepare functional magnetized graphene load thermal conductive materials in the study of high thermal conductivity of graphene. Through the negative charge packaging polymer, it is attached to the graphene surface, and combined with the positively charged polyelectrolyte under the action of electrostatic force, and the negatively charged nanoparticles $\mathrm{Fe}_{3} \mathrm{O}_{4}$ are adsorbed on the surface by the positive charge, so as to realize the adsorption of nanoparticles and graphene together. Finally, graphene- $\mathrm{Fe}_{3} \mathrm{O}_{4}$ is combined with epoxy resin in the external magnetic field environment, which can control the order of graphene. The thermal conductivity of magnetized graphene is more than twice that of disordered graphene when the thermal conductive material is $1 \%$. In the test and analysis of load operation, it can be found that the CPI temperature of randomly filled with $2 \%$ graphene is about $5{ }^{\circ} \mathrm{C}$ lower than that without filling material after $15 \mathrm{~h}$ of operation, and the temperature of $\mathrm{CPU}$ can be decreased by $10{ }^{\circ} \mathrm{C}$ with $1 \%$ graphene after magnetization. Controlling graphene in polymer matrix can improve the thermal conductivity of the composites. Anisotropic thermal conductivity in the process of transmission, it is through a single channel to achieve rapid transfer, not to the surrounding. Under this characteristic, the thermal conductivity of graphene perfluoroalkoxy nanocomposites can reach more than $25 \mathrm{~W} /(\mathrm{m} \cdot \mathrm{K})$ in practical application, which can be used in light-emitting diodes. In order to further study the relationship between thermal conductivity and heat transfer direction of different graphene composites, the thermal conductivity of graphene / copper composites can be analyzed by vacuum filtration and discharge plasma. When the content of graphene reaches $30 \%$, the maximum value of thermal conductivity is $458 \mathrm{w} /(\mathrm{m} \cdot \mathrm{K})$. There are some differences in the heat transfer direction and the different angle of graphene nanoflakes. The thermal conductivity of $30 \%$ graphene nanoflakes can reach $826 \mathrm{w} /$ $(\mathrm{m} \bullet \mathrm{K})$ In a fully aligned state. In practical application, the epoxy resin material can be added with carbon nano meter, and its thermal conductivity is relatively high. On the basis of realizing thermal resistance control, it can provide materials for heat dissipation of electronic components.

\subsection{Application of Graphene Oxide Composites}

The compatibility between graphene and polymer matrix is poor, and it is easy to agglomerate. However, there are more functional groups on the surface of graphene oxide, and it has high dispersion in aqueous solution. Moreover, functional groups can be modified to improve its compatibility with polymer substrate, reduce agglomeration, and play a positive role in improving the heat dissipation performance of packaged electronic 
components. In practical application, the thermal conductivity of graphene oxide composite is $0.6 \mathrm{~W} /(\mathrm{m} \cdot \mathrm{K})$ in the state of $25 \%$ graphene. In addition, in the research and analysis of graphene oxide, when graphene oxide is added into the original epoxy slurry, and its volume fraction changes to $7 \%$, the thermal conductivity is the maximum state. In the preparation of graphene oxide composites, graphene oxide can be added into epoxy resin before the curing agent is mixed. In addition, it can be prepared by polycondensation between graphene oxide and curing agent, and then mixed with epoxy resin after precipitation of suspension colloid. In the actual operation process, adding $3 \%$ graphene and its oxide, its thermal conductivity can be increased to $175 \%$ in the study of its thermal conductivity. In the state of high temperature operation, the service life and luminous stability of LED chips are relatively reduced. Therefore, after using graphene oxide, its heat dissipation effect is more obvious. Graphene oxide is easy to be scattered in practical application, which has a direct impact on its thermal conductivity. If graphene oxide is used as a filler to enhance thermal conductivity, its heat conduction enhancement effect is not obvious. However, under the condition of polycondensation, the oxygen-containing functional groups and the substrate materials can form covalent bonds to connect, thus reducing the scattering problem of graphene oxide, thus further improving the thermal conductivity of the composite. In the research and analysis of graphene oxide, its thermal conductivity is directly related to the practical application, thermal conductivity and graphene content of graphene oxide. Under the premise of controlling the thermal conductivity of graphene oxide, graphene oxide / epoxy resin composite thermal conductivity material can be prepared. In practical application, the thermal conductivity of graphene oxide is $45 \%$ higher than that of pure copper.

\section{CONCLUSION}

To sum up, in the research on the preparation and practical application of graphene, the practical application effect can be improved by the thermal conductivity of graphene. In practical application, graphene composites can improve the thermal conductivity of graphene composites under the action of magnetic field by using its high plane thermal conductivity. In practical application, graphene can be combined with other materials with different thermal conductivity, and its thermal conductivity can be further improved, which has a positive role in improving the application range of graphene. In the research on the application of minority layer graphene, graphene oxide and graphene composites, their thermal conductivity is different, and their application fields and thermal conductivity effect are also different. On the basis of optimizing the thermal conductivity control of graphene composites, the practical application effect of graphene can be further improved. The preparation of coal based graphene can reduce the difficulty of preparation of coal based graphene through heat treatment. On the basis of controlling the content of graphene oxide, the quality of coal based graphene can be improved. In the research and analysis of the thermal conductivity mechanism of coal based graphene, different phonons have different heat transfer effects in different environments. From this perspective, optimizing the practical application process of graphene will play a positive role in improving the practical application effect of coal based graphene.

\section{REFERENCE}

[1] Rui-Qing Li, Yue-Gang Tang, Xuan Xun, Jiang-Tao Fan, Qi-Li Che. Research Progress on Raw Materials and Preparation Technology of Coal based Graphene. Coal Geology \& Exploration. 2020;48(05): 1-15.

[2] Yun-Fei Yan, Wei Gao, Zhong-Qing Yang, Li, Zhang, Jing-Yu Ran. Coal base New Materials-Progress in Preparation of Coal based Graphene and Its Application in Thermal Conductivity. Journal of China Coal Society. 2020;45(01): 443-454.

[3] Ya-Ting Zhang, Jing-Jing Han, Kai-Bo Zhang, Jing Zhang, Ke-Ke Li, Kai-Li Jia, An-Nan Xu. Preparation of sulfur doped graphene quantum dots from coal and its detection of $\mathrm{Ag} \sim$. Carbon Techniques. 2019; 38(05): 39-42.

[4] Yue-Gang Tang, Jing-Jie Xu, Xuan Xun, Shao-Qing Wang, Peng-Xiang Chen. Preparation and Spectral Characterization of Anthracite based Graphene of Yunnan Xiaofalu. Journal of China Coal Society. 2020; 
45(02): 740-748.

[5] Hai-Yan Liu, Wen-Xue Lu, Yong-Guo Wu, Mei Han, Jin-Zhong Zuo, Xue-Xiang Deng, Zhen-Hua Wang. Research Progress of Coal based Graphene and Its Composites. Coal Processing \& Comprehensive Utilization. 2019; (05): 84-87.

[6] Tie-Hu Li, Wei-Jie Liang, Hao Li. Research Progress of Coal based Graphene modified Resin Thermal Interface Materials. Coal Conversion. 2019; 42(01): 1-8.

[7] Wen-Qian Luo. Application of Graphene in Heat Conduction and Heat Dissipation. Advanced Materials Industry. 2018; (11): 35-37.

[8] Chun-Bao Zhao, Zhen Liu. Preparation and Electrochemical Properties of Coal based Graphene. Chemical Engineer. 2017; 31(10): 14-16+22.

[9] Ya-Ting Zhang, Shao-Zhao Ren, Jing-Kai Li, Ke-Ke Li, Yong-Qiang Dang, Guo-Yang Liu, Jie-Shan Qiu. Preparation and Electrochemical Properties of PANI / Coal based Graphene Macro Composites. Journal of Chemical Industry and Engineering(China). 2017; 68(11): 4316-4322.

[10] Hui-Hui Zeng, Gui-Yun Yi, Bao-Lin Xing, Guang-Xu Huang, Lun-Jian Shen, Chuan-Xiang Zhang, Bing Xu, You-Heng Yao, Qing-Shan Zhang, Jie Li. Coal based Graphene/Preparation and Photocatalytic Properties of Composite Materials of TiO_2. Chemical Industry and Engineering Progress. 2017; 36(07): 2568-2576. 